\title{
Cambio teórico y progreso en bioquímica*
}

\author{
Lucía Federico y Jorge Paruelo**
}

\begin{abstract}
Resumen
El progreso científico es uno de los temas más tratados por la filosofía de la ciencia. Actualmente el área ofrece un abanico de modelos de progreso científico para elegir a la hora de abordar los procesos concretos que se dieron en una disciplina particular de la ciencia. En el presente artículo nos proponemos analizar la noción de cambio teórico en bioquímica, pero trasladable a la biología y ciencias biomédicas por hacer uso del mismo conjunto de teorías, bajo una de las perspectivas de progreso científico, la de P. Kitcher, que creemos es una de las más adecuadas para arrojar claridad a la discusión del caso.

Palabras clave: Progreso cientifico, Cambio, Teoría, Explicación, Bioquímica.
\end{abstract}

\begin{abstract}
Scientific progress is one of the most popular topics in philosophy of science. Currently, the area offers a range of models to choose scientific progress, when addressing the specific processes that occurred in a particular discipline of science. In this article we analyze the notion of theoretical change in biochemistry, but translatable to biology and biomedical sciences by making use of the pull of theories, under one of the prospects of scientific progress, P. Kitcher, which we believe is one of the best to shed clarity to the discussion of the case.
\end{abstract}

Keywords: Scientific progress, Change, Theory, Explanation, biochemistry.

\footnotetext{
* Recibido: mayo 2016. Aceptado: junio 2016.

** Universidad de Buenos Aires. Buenos Aires, Argentina. Email: luciafed@hotmail.com / 


\section{Introducción}

En la sociedad actual está ampliamente aceptado que la ciencia progresa casi en forma constante. Hay discusiones acaloradas respecto de si algunas aplicaciones tecnológicas constituyen un avance o si, por el contrario, indican que la ciencia actual genera más problemas de los que resuelve y por lo tanto es dudoso el progreso mencionado. Se puede argumentar que esta discusión apunta más a los usos de la ciencia que al conocimiento que ella genera. Independientemente de qué posición se adopte respecto de esta última discusión, el conocimiento que brinda la ciencia, más allá de sus usos, ha tenido claros progresos a lo largo de su historia y es lo que rescata la frase inicial.

El término "progreso científico", sin embargo, requiere de algún tipo de análisis y elucidación. Distintas corrientes en filosofía de la ciencia han adoptado diferentes posiciones acerca de lo que entienden por tal término, lo que ha llevado a multiplicar conceptos para dar cuenta de los distintos tipos de progreso científico y de sus características: se habla de "progreso acumulativo", "progreso por rupturas", "progreso hacia", "progreso desde", "progreso instrumental", etc.

No pretendemos aquí elucidar el término, pero es necesario fijar algunas posiciones en relación al progreso científico. En primer lugar, todo progreso involucra algún cambio. Es imposible pensar que alguna rama de la ciencia progresa si no se identifica al menos algún tipo de cambio en su desarrollo. Pero no todo cambio involucra progreso. La identificación de cambios es previa a la determinación de si dicho cambio implica progreso. Por esta razón en este trabajo se propone un análisis de los cambios en ciencia y sus características para luego determinar si resultan progresivos o no.

La identificación de un cambio como progresivo se hace sobre la base de una comparación entre estados, de un estado que puede ser previo o posterior a otro. Se puede pensar que hay progreso hacia determinado punto (a la verdad, por ejemplo) o se puede hacer referencia a estados previos. Se adoptará en lo que sigue la idea de que el cambio progresivo se mide en función de las condiciones precedentes inmediatas, es decir, en relación al conocimiento inmediatamente previo del que se disponía en el momento del cambio. Aclararemos esto más adelante.

En el presente trabajo mostramos un caso de cambio científico en bioquímica y analizamos si constituye un cambio progresivo. En la sección 2 distinguimos algunos tipos de cambio científico. En la sección 3 delimitamos el ejemplo de cambio bioquímico y presentamos algunas de sus características. Finalmente, en la sección 4 analizamos si dicho cambio resulta progresivo sobre la base de algún criterio de progreso. 


\section{Cambios científicos}

La discusión que se da alrededor del cambio teórico es una de las tantas problemáticas que, sin duda, se aborda desde el marco conceptual aportado por la filosofía diacrónica de la ciencia. Hay coincidencia en que fue a partir de la llamada "revuelta historicista" que se enfatizó la necesidad de una filosofía de perspectiva diacrónica, además de la clásica perspectiva sincrónica disponible en la época. Surgieron así un conjunto de conceptos nuevos, como la noción de paradigma o matriz disciplinar de Kuhn, los programas de investigación de Lakatos, la red teórica estructuralista, entre otros, que proponían una nueva forma de pensar las teorías como entidades que evolucionan en el tiempo.

Aunque la introducción de la noción de "revolución científica" (y todo lo que ello conlleva) resultó revolucionaría en el ámbito de la filosofía de la ciencia, actualmente la mayoría de los filósofos coinciden en que el termino carece de precisión y que incluso (salvo para algunos casos) resulta equívoco (Moulines y Diéz, 1997).

Podemos distinguir (Miguel, Paruelo y Pissinis, 2002) cinco tipos de cambio teórico asociados cada uno de ellos con el cambio conceptual, introducido para la resolución de un problema en relación a un marco teórico dado. Los tipos propuestos son:

I. cambio que no involucra ninguna novedad inesperada, y no altera la clasificación de entidades y procesos que, según nuestras mejores teorías vigentes, pueblan el mundo. Un ejemplo de este cambio es dar cuenta del movimiento de un péndulo a partir de las leyes de la mecánica;

II. cambio que involucra una nueva entidad de un tipo conocido. Por ejemplo, la resolución del movimiento de Urano asociada al descubrimiento de Neptuno;

III. cambio que involucra una modificación en la ontología presupuesta en la teoría pero que es compatible en algún grado con las leyes de la misma. Un ejemplo de esto es el caso del descubrimiento del neutrino. La introducción de este nuevo tipo de partícula obligó a cambiar la taxonomía de partículas admitidas por la teoría. Este tipo de cambio puede traer consigo alguna modificación de leyes, sea por agregado o por necesidades de refinamiento;

IV. cambio que involucra una modificación en la ontología que introduce alguna incompatibilidad con las leyes de la teoría. Este cambio conduce a un cambio de marco conceptual.

V. cambio de marco conceptual que no tienen su origen en un cambio ontológico puntual. En estos casos es notable la ruptura tanto en las leyes como en la clasificación, pero en el sentido de que los procesos y entidades que pueblan el mundo pasan a ser descritos, definidos y 
comprendidos de una manera diferente. Este caso es el más difundido como revolución científica y remite a un cambio global del marco conceptual.

Los dos primeros tipos de cambio se asocian a lo que Kuhn llamó "resolución de enigmas" en un período de ciencia normal. Los dos últimos son cambios revolucionarios ya que involucran cambio de marco. El tercero es un caso de decisión controvertida respecto de su carácter de revolucionario y no es propósito de este trabajo fijar una postura en esa discusión. En cualquier caso, la numeración propuesta sí indica un orden creciente en la magnitud del cambio.

En la mayor parte de los ejemplos históricos científicos, el cambio abrupto no se detecta, en su lugar se presentan cambios más atenuados. Dicho de otro modo, si la numeración presentada indica un orden creciente de magnitud de tipos de cambio, también estaría insinuando uno decreciente en la frecuencia de casos históricos.

Si sumamos a la clasificación anterior un enfoque más detallado desde las leyes involucradas, tenemos que los cambios pueden ser tales que la teoría nueva requiera modificaciones en leyes de distinto grado de generalidad. Desde este punto de vista, en los tipos de cambio I y II mencionados antes no habría cambio teórico. En los de tipo III puede darse el caso de que se incorporen leyes o restricciones nómicas de bajo nivel de generalidad o que se introduzcan leyes de mayor nivel de la que se obtengan las de menor nivel. En cualquier caso, las leyes previas al cambio no dejan de tener validez ya que son compatibles con las de la teoría después del cambio. En este tipo de cambio, los casos de aplicación de la teoría previa siguen siendo casos de aplicación de la posterior pero esta última puede tener casos no contemplados en la primera. Cabe ampliar este tipo de casos sumando aquellos en los que no hay una modificación significativa de la ontología pero se amplía el alcance de la teoría. En este último tipo de cambio (Moulines y Diéz, 1997: 450), la teoría anterior es suplantada sólo en parte por la teoría posterior; muchos de los conceptos de la primera persisten (iguales o con modificaciones semánticas leves) en la segunda teoría, igual que algunos principios o leyes y aplicaciones o casos paradigmáticos; la teoría anterior es reinterpretada como un caso "especial" de la posterior o como una "aproximación" de la segunda. A su vez, en el plano sociológico, la comunidad científica no queda dividida en dos, más bien una parte de ella, aunque adherente a la nueva teoría, sigue trabajando con la antigua, sea por fines didácticos, sea para resolver problemas de un ámbito restringido o en busca de aplicaciones tecnológicas concretas.

En los casos de cambio de tipos IV y V nos encontraríamos con cambios semánticos y de leyes significativos que involucrarían inconmensurabilidad.

En resumidas cuentas podemos decir que hay tres tipos de cambio relevantes para lo que nos interesa analizar: el cambio intrateórico (I y II), el 
cambio interteórico en el que la teoría nueva 'suplanta' a la previa (IV y V) y el interteórico en el que la teoría posterior 'incorpora' a la previa (uno de los tipificados como III).

Un tercer parámetro a tener en cuenta para el análisis del cambio teórico es el conjunto de aplicaciones de la teoría. En los casos IV y V suele modificarse el conjunto de aplicaciones de tal manera que algunos casos pierden sentido y se establecen otros nuevos. En el cambio de tipo III, puede ampliarse dicho conjunto o mantenerse igual, pero modificando el conjunto de elementos teóricos empleados para dar cuenta de él.

\section{Un ejemplo bioquímico de cambio interteórico}

En estos tiempos que corren difícilmente nos encontremos con alguien que no haya hecho uso del ibuprofeno para aplacar algún dolor, sea este de cabeza, muscular o de otra índole. Penosamente, quién no ha escuchado alguna vez noticias de decesos, generalmente hogareños, producto de ese gas inoloro que causa un silencioso envenenamiento, el monóxido de carbono. Y si bien, estos dos sucesos resultan familiares, seguramente al lector no entrenado en esta área de conocimiento le puede resultar difícil vincularlos. Es posible mostrar, sin embargo, que pese a todo son factibles de vinculación producto de una elucidación con el instrumental adecuado.

Antes de avanzar en tal tarea, conviene hacer un primer acercamiento analítico presentando los casos en lenguaje científico.

El ibuprofeno es un fármaco que focaliza su acción en una enzima que poseen algunas células de nuestro cuerpo: la ciclooxigenasa (COX). Las enzimas son macromoléculas que catalizan reacciones químicas dentro y fuera de las células. En los procesos de inflamación actúa una sustancia llamada "prostaglandina" que se produce a partir de la oxidación del ácido graso araquidónico. Esta oxidación ocurre en el centro catalítico de la ciclooxigenasa. El ibuprofeno compite con el ácido araquidónico por 'ocupar' el centro catalítico de la enzima y de esa manera inhibe la producción de prostaglandina disminuyendo la inflamación y el dolor.

Por otro lado, entre los causales de muerte más comunes en el ámbito doméstico (con una incidencia de un 50\%) se encuentra la intoxicación por monóxido de carbono (ICO), producto de una combustión incompleta, al no formarse dióxido de carbono $\left(\mathrm{CO}_{2}\right)$ por falta de oxígeno. La hemoglobina, macromolécula encargada del trasporte de oxigeno a los distintos tejidos del cuerpo, es capaz de ligar el monóxido de la misma forma que lo hace con el oxígeno. Ambas moléculas son semejantes, pero la afinidad del CO por los centros de unión de la hemoglobina es mayor que la del oxígeno. En presencia de ambas moléculas el CO gana la competencia por los sitios de unión y la hemoglobina termina trasportando una sustancia de elevada toxicidad a los tejidos. 
La explicación del modo como operan el ibuprofeno y el monóxido nos da la pauta del vínculo que hay entre dos fenómenos de consecuencias tan disímiles. En ambos casos se encuentra implicada una macromolécula, una proteína, cuya función normal se ve afectada por sustancias que compiten con sus habituales sustratos (las sustancias con la que cumplen su acción fisiológica): ibuprofeno y ácido araquidónico compiten por el lugar en la ciclooxigenasa mientras que oxígeno y monóxido de carbono compiten por el suyo en la hemoglobina (el 'triunfo' del ibuprofeno reduce la inflamación mientras que el del monóxido conduce a la muerte). Con esta caracterización un lector avezado, con conocimiento en biología, señalará que actualmente existe un conjunto de "teorías" (también llamados en el ámbito científico "modelos" o "ecuaciones") que dan cuenta de los patrones de actividad de las proteínas funcionales: enzimas, trasportadores, receptores, canales, etc.

De forma sucinta, las primeras propuestas datan de 1904, a partir de los estudios de Niels Bohr sobre la afinidad de la hemoglobina por el oxígeno, hoy día el "efecto Bhor"; en 1910 los estudios de Archibald Hill sobre la cooperatividad (o curva de actividad con forma sigmoidea) de la misma proteína, sintetizados en "la ecuación de Hill"; pasando por la teoría enzimática de Michaelis-Menten de 1913; "la ecuación de Adair" de 1924 para la cooperatividad; a las más resonantes: la de Jack Monod y colaboradores del '65 y la de Daniel Koshland un año después; hasta las modernas de múltiples estados conformacionales. Y si bien todas ellas constituyen parte fundamental de la bioquímica, la farmacología, la biología molecular y la incipiente biología de sistemas, poco se ha explorado las relaciones entre las propuestas. A primera vista la impresión que da el revisar los textos especializados es que todas son utilizadas, incluso las propuestas más antiguas, aunque aggiornadas.

Del total de los modelos de cooperatividad actualmente aceptados, los más populares son el de A. Hill y el modelo alostérico de J. Monod, Jeffries Wyman y Jean-Pierre Changeux, o modelo MWC, pero es este último el que se dice que presenta un mecanismo explicativo (Kesse y Ben-Tal, 2011:168). Veremos si por medio del mencionado modelo podemos vincular la acción del ibuprofeno y del CO bajo un mismo patrón de explicación, pues al menos sabemos que la hemoglobina presenta cooperatividad.

\subsection{El modelo alostérico}

La novedad del modelo se debió al planteo, como conceptos explicativos o novedosos, de dos "estados conformacionales" para una misma proteína ${ }^{1}$. Esta propuesta resultó bastante audaz en su época pues no había prueba empírica que lo sustentara. Cada estado conformacional, según el modelo, conlleva un tipo de estructura tridimensional particular y una actividad biológica asociada: el estado "tenso" (T) con baja actividad y el estado "relajado" (R) con alta actividad, representados por $\tau$ y $\mathrm{r}$ respectivamente. Cada estado con-

\footnotetext{
${ }^{1}$ La reconstrucción de la teoría completa puede verse en Alleva, Diez y Federico (2012).
} 
formacional, a su vez, se une a cada tipo de ligando con una afinidad particular, representadas por $\kappa_{\Psi \tau} \mathrm{y} \kappa_{\Psi r}$ respectivamente. El estado conformacional en que se puede encontrar una proteína de la población en un momento particular, como si se le sacáramos una foto, es representado por $\zeta$.

Los tipos de sistemas biológicos a los que se pretende aplicar el modelo son poblaciones de proteínas llamadas oligoméricas por tener más de una subunidad, cada una de las cuales tiene un sitio de unión para cada tipo de ligando. El número de subunidades está representado por $\rho$. Los ligandos pueden ser su sustrato, un activador o un inhibidor (moduladores) de la actividad, representados por $S, A, I$ respectivamente. En cada sistema puede estar presente el sustrato o el sustrato acompañado por uno o dos moduladores (activadores e inhibidores). Estos sistemas a su vez presentan en cada momento una actividad biológica particular, cooperativa o no, representada por $\delta$, en función de la cantidad de ligando unido a las proteínas en cada momento, representado por $\left|\Psi_{t}\right|$.

En la publicación de 1965, encontramos que los autores formulan tres restricciones nómicas para dar cuenta de los sistemas anteriormente caracterizados. La primera asume que en ausencia de ligando los estados $\mathrm{T}$ y $\mathrm{R}$ de una población están en equilibrio: el total de proteínas en estado $\mathrm{T}$ y el total en estado R son constantes. El valor de la relación entre los estados es conocido como "constante alostérica" $\left(l_{0}\right)$. La segunda restricción asume que sólo las formas no unidas a ligando, en cualquiera de los estados, pueden cambiar de conformación. Ese cambio fue denominado "transición alostérica" (de todas las subunidades juntas y por eso "concertado"). Una vez unida la proteína a un ligando queda "fijada" en una conformación no pudiendo producirse una nueva transición alostérica. Por último, la tercera ley vincula el cambio conformacional con la actividad biológica de la siguiente manera:

$$
\frac{\delta_{(t)}=\left[\iota_{0 t} \cdot\left(1+\varepsilon_{I t t}\right)^{\rho o /} /\left(1+\varepsilon_{A r t}\right)^{\rho o}\right] \cdot \varepsilon_{s t t^{*}}\left(1+\varepsilon_{s t t}\right)^{\rho o-1}+\varepsilon_{s t t^{*}}\left(1+\varepsilon_{s t r}\right)^{\rho o-1}}{\left[\iota_{0 t} \cdot\left(1+\varepsilon_{I t t}\right)^{\rho o /} /\left(1+\varepsilon_{A r t}\right)^{\rho o}\right] \cdot\left(1+\varepsilon_{s t t}\right)^{\rho o}+\left(1+\varepsilon_{s t t}\right)^{\rho o}}
$$

DfAux: $\varepsilon_{\Psi t}=\left|\Psi_{t}\right| / \kappa_{\Psi}$ (es la cantidad de ligando "normalizado" unido a las proteínas en un estado conformacional).

Esta ley establece que el grado de actividad está dado por la proporción de proteínas unidas (en ambos estados conformacionales) sobre el total de uniones posibles en la población.

Volviendo a nuestros dos casos de interés, nos preguntaremos ¿cómo son nuestras proteínas? ¿son, junto a sus ligandos, sistemas factibles de ser explicados por este modelo?

La hemoglobina es un tetrámero formado por cuatro subunidades unidas entre si. Cada subunidad posee un grupo "hemo", una estructura anular que 
contiene un átomo de hierro, capaz de unir un oxigeno. Además, la unión de un oxígeno incrementa la probabilidad de unión de otro oxigeno en la subunidad vecina, el fenómeno que llamamos "cooperatividad".

La estructura de COX no es habitual en la bibliografía, pero encontramos su descripción en artículos especializados. Se trata de un dímero que, en cada subunidad, posee un sitio activo ciclooxigenasa y otro peroxidasa (que activa el grupo hemo que participa en la reacción de la ciclooxigenasa). Al igual que la hemoglobína posee más de una subunidad, contiene grupos hemo y presenta cooperatividad (WWProtein Data Bank, 2016); (Zou et al, 2012).

En principio, las características de ambas proteínas indican que los sistemas que conforman podrían ser explicados por MWC. Si agregamos los ligandos, el ibuprofeno y el monóxido, que son dos inhibidores, el análisis nos lleva a los sistemas dónde aparece la acción de los moduladores.

Los moduladores modifican el patrón de actividad, acelerándolo o inhibiéndolo. En el modelo ambos ligandos actúan ejerciendo su acción mediante su unión en un sitio específico, así, cada subunidad, además del sitio catalítico (donde se une el sustrato), suele presentar un sitio de unión para cada tipo de modulador (el "alostérico"). Cada modulador en el modelo se une a un estado conformacional particular. El estado R tiene alta afinidad por el activador mientras que el estado $\mathrm{T}$ lo tiene por el inhibidor.

Ahora bien, la asunción de estos supuestos nos presenta un fuerte impedimento al tratar de contemplar nuestros ejemplos como aplicaciones de este modelo: la tercera restricción nómica no incluye otra clase de inhibición que aquella que es producto de la unión a un sitio específico distinto al catalítico, a diferencia de nuestros dos inhibidores que compiten por unirse al mismo sitio que el sustrato. Y con ello se genera un problema adicional: si el ibuprofeno y el monóxido compiten por la unión a tal sitio, lo harán al igual que el sustrato, predominantemente cuando la proteína esté en estado $\mathrm{R}$, con el centro catalítico "expuesto". Pero el inhibidor modulador lo hace uniéndose a una región expuesta en el estado $\mathrm{T}$, estabilizando la proteína en su forma poco activa.

Por tanto, no nos queda más que descartar ICO y COX como aplicaciones de este modelo y buscar otra alternativa. Nuestro segundo candidato es la teoría michaeliana, sin duda una de las propuestas más enseñadas en la biología, que contempla la inhibición competitiva.

\subsection{La teoría de Michaelis y Menten}

Los estudios acerca de las curvas de actividad de las enzimas llevaron a la conformación de una nueva subdisciplina: la enzimología. La propuesta desarrollada por Leonor Michaelis y Maud Menten en 1913 y cristalizada con los aportes de George E. Briggs y John B.S. Haldane en 1925, describe cómo varía la velocidad de catálisis a medida que varía la cantidad de ligando. 
Las enzimas poseen una afinidad particularmente alta por su sustrato y poca por otras sustancias, por lo cual se las considera moléculas "específicas". Este valor de la afinidad es representado mediante la "constante de afinidad": $\kappa_{S}, \kappa_{I}$ para el ligando sustrato $(S)$ y el inhibidor $(I)$ respectivamente.

La restricción nómica de la propuesta michaeliana, según nuestro análisis, conecta la actividad biológica con el grado de afinidad de las enzimas a los ligandos, sustrato e inhibidores. Esta regularidad establece que en cada momento la actividad coincide cuantitativamente con la proporción de enzimas de la población cuyos sitios catalíticos se encuentran ocupados por ligando, sobre el total de uniones posibles en la población. Formalmente:

$$
\delta_{(t)}=\varepsilon_{s t} /\left(1+\varepsilon_{i^{\prime} t}\right)+\varepsilon_{s t}\left(1+\varepsilon_{i^{\prime \prime} t}\right) \text { donde DfAux: }:^{2} \varepsilon_{\Psi_{t}}=\left|\Psi_{t}\right| / \kappa_{\Psi}
$$

(es la cantidad de ligando "normalizado")

Cómo se establece en la formulación, los inhibidores pueden ser de dos tipos: competitivos $\left(I^{\prime}\right)$ o no competitivos $\left(I^{\prime \prime}\right)$. La diferencia está en si la sustancia compite con el sustrato por unirse al sitio catalítico o si la sustancia se une al mismo tiempo que el sustrato en otro sitio inespecífico.

Como señalamos, esta teoría da cuenta de la actividad de las proteínas incluso en presencia de inhibidores por lo cual parece ser una buena candidata para explicar la acción del ibuprofeno y del envenenamiento por monóxido de carbono, dos inhibidores competitivos. Aunque el COX sea una enzima y la hemoglobina un transportador, el usuario de la teoría en principio no hace diferencia.

Sin embargo, la teoría fue desarrollada para un tipo particular de proteínas, pues asume que cada enzima sólo tiene un sitio de unión o "centro catalítico". Lamentablemente la restricción nómica sólo contempla una unión por molécula, e ignora así el efecto cooperativo. Esto descarta al sistema hemoglobina y la ciclooxigenasa como aplicaciones de la teoría.

En este punto es donde quizás el lector con conocimiento científico quede un tanto alarmado pues, aunque haga uso de su memoria, caerá en la pregunta del lector lego: ¿qué teoría los explica?

\subsection{La urgencia de una nueva teoría}

En la bibliografía específica del área se enfatiza que la explicación de estos fenómenos la provee el modelo de Monod. Ejemplo de ello es la forma como se expone el caso de la enzima (alostérica) ACTasa en presencia del inhibidor (competitivo) malato, que es presentada bajo el título "Efectos de la inhibición competitiva", alertando al final de la sección que "modelos especiales han sido diseñados para dar cuenta de esta clase de comportamiento"

\footnotetext{
${ }^{2} \varepsilon_{\Psi_{\tau}}$ se corresponde con la cantidad de uniones de ligando $\Psi$ a la enzima $e$ en el momento $t$, dividido la constante de disociación $k$ para el ligando correspondiente.
} 
(Cantor y Schimmel, 1980: 945-945), pero curiosamente a esto le sigue la exposición del "Modelo MWC". Y, como es posible mostrar, dicho modelo no puede dar cuenta ni de éste, ni del ibuprofeno ni del envenenamiento por monóxido

Sin embargo, estamos seguros de que hay una teoría implícita de mayor grado de generalidad que las anteriores que permite explicar tanto los sistemas descritos por la teoría alosterica como por la michaeliana. Es decir, poseería un enorme poder de unificación (Diez, 2002) y por alguna razón ha pasado desapercibida.

El sustento formal de tal hipótesis lo constituye el hecho de que, conociendo la forma lógica de las leyes y mediante el instrumental adecuado, es factible arribar a la ecuación general de una teoría que integre todos los tipos de ligandos mencionados, como también la posibilidad de cambio conformacional. A continuación procedemos a mostrarlo.

Si mantenemos inalteradas las dos primeras leyes del modelo MWC en esta nueva teoría, que llamaremos Monod-Michaelis-Menten (TMMM), y remplazamos la tercera de sus leyes por una que también contemple los inhibidores no moduladores, esta nueva ley adoptará la siguiente forma lógica:

$$
\frac{\delta_{(t)}=\left[\left[\iota_{0 t}\left(1+\varepsilon_{I t t}\right)^{\rho o /} /\left(1+\varepsilon_{A r t}\right)^{\rho o}\right] \cdot \varepsilon_{s t t^{\prime}}\left(1+\varepsilon_{s t t}\right)^{\rho o-1}+\varepsilon_{s t t^{\prime}}\left[\left(1+\varepsilon_{i^{\prime} t}\right)+\varepsilon_{s t r}\left(1+\varepsilon_{i^{\prime \prime} t}\right)\right]^{\rho o-1}\right]}{\left[\left[\iota_{0 \tau} \cdot\left(1+\varepsilon_{I t t}\right)^{\rho o /} /\left(1+\varepsilon_{A r t}\right)^{\rho o}\right] \cdot\left(1+\varepsilon_{s t t}\right)^{\rho o}+\left[\left(1+\varepsilon_{i^{\prime} t}\right)+\varepsilon_{s t t} \cdot\left(1+\varepsilon_{i^{\prime \prime} t}\right)\right]^{\rho o}\right]}
$$

DfAux: $\varepsilon_{\Psi t}=\left|\Psi_{t}\right| / \kappa_{\Psi}$ (es la cantidad de ligando "normalizado" en un estado conformacional)

La misma establece que el grado de actividad biológica (para una población de proteínas con ligando sustrato, moduladores e inhibidores no-moduladores) en cada momento, coincide con la proporción de subunidades unidas a ligando (en ambos estados conformacionales y según su respectiva afinidad) sobre el total de uniones posibles.

¿Pero cómo distinguir, si la función y la teoría no son un mero artilugio matemático, un constructo obtenido de la conjunción de dos fórmulas o si realmente representa una teoría fáctica?

Es claro que para que se dé esto último, la nueva formulación tiene que cumplir un conjunto de restricciones empíricas propias de los fenómenos biológicamente relevantes que pretende explicar. Pero ¿lo hace?

Si uno mira en detalle los componentes de la ley verá que no es caprichosa la forma en que cada ligando, con su constante de afinidad, se une a una conformación específica de las proteínas de la población. Tal disposición no es fruto de un proceso de encadenamientos deductivos, todo lo contrario, surge de los datos empíricos obtenidos a partir de la bibliografía del área. Podríamos decir que la formulación tiene aún el estatus de "una hipótesis a 
contrastar". Pues bien, si se nos concede lo hasta aquí dicho nos resta mostrar que nuestra ley, en el marco de la teoría, es fructífera a la hora de capturar los fenómenos biológicos mencionados y así fortalecer nuestra intuición de que la reconstrucción refleja la teoría implícita que buscamos. Demostraremos por tanto como esta nueva propuesta captura los sistemas ICO y COX.

Para ello expondremos cómo dar cuenta de los sistemas que aquí nos ocupan, empezando por la intoxicación por monóxido de carbono, a partir de las leyes de TMMM. Para lograrlo hace falta introducir una serie de (nuevas) restricciones adicionales sobre las leyes generales. Operando de esta manera es factible "llegar" hasta las representaciones matemáticas de las leyes (específicas) que los capturan. En terminología kuhniana, es encontrar la nueva forma que adoptará la generalización simbólica para dar cuenta de un caso concreto de aplicación.

En nuestro sistema encontramos una proteína con más de una subunidad que presentan cooperatividad entre sí, es decir que es una proteína del tipo alostérica. La primera restricción que se introduce a las leyes generales para capturar este tipo de comportamiento de la población es que, en ausencia de ligando, hay menor cantidad de proteína en estado $\mathrm{T}$ que en estado $\mathrm{R}$ y que la afinidad por el sustrato es mucho mayor para el estado R que para $\mathrm{T}\left(\kappa_{S_{\tau}}<<\right.$ $\kappa_{S r}$ ). Bajo estas restricciones la actividad biológica (para proteínas con cooperatividad) adquiere la siguiente forma:

$$
\begin{gathered}
\delta_{(t)}=\varepsilon_{s t t} \cdot\left[\left(1+\varepsilon_{i^{\prime} t}\right)+\varepsilon_{s r t}\left(1+\varepsilon_{i^{\prime \prime} t}\right)\right]^{\rho o-1} \div[ \\
{\left[\iota_{0 t} \cdot\left(1+\varepsilon_{I t t}\right)^{\rho o} /\left(1+\varepsilon_{A r t}\right)^{\rho o}\right]+\left[\left(1+\varepsilon_{i^{\prime} t}\right)+\right.} \\
\left.\varepsilon_{s r t} \cdot\left(1+\varepsilon_{i^{\prime \prime} t}\right)\right]^{\rho o}
\end{gathered}
$$

Si se añade además que no hay presencia de moduladores alostéricos (ni activadores ni inhibidores) en el sistema $\left(\varepsilon_{A r t=} \varepsilon_{\mathrm{It} t=} 0\right)$ y todas las subunidades de la proteína se pueden unir o al sustrato o a algún inhibidor no-modulador, la forma que adquiere ahora la ecuación de actividad biológica es:

$$
\delta_{(t)}=\varepsilon_{s t t} \cdot\left[\left(1+\varepsilon_{i^{\prime} t}\right)+\varepsilon_{s r t}\left(1+\varepsilon_{i^{\prime \prime} t}\right)\right]^{\rho o-1} \div \iota_{0 t}+\left[\left(1+\varepsilon_{i^{\prime} t}\right)+\varepsilon_{s r t} \cdot\left(1+\varepsilon_{i^{\prime \prime} t}\right)\right]^{\rho o}
$$

Por último, si agregamos a esta ley (que captura los sistemas alostéricos parecidos a los llamados "homotrópicos" del modelo MWC) la restricción de ausencia de inhibidor no competitivo $\left(\varepsilon_{i^{\prime \prime} t}=0\right)$ y teniendo en cuenta que el inhibidor competitivo tiene afinidad por el estado conformacional R (como el sustrato), la actividad biológica toma el siguiente valor específico:

$$
\delta_{(t)}=\varepsilon_{s t t} \cdot\left[\left(1+\varepsilon_{i^{\prime} t}\right)+\varepsilon_{s t t}\right]^{\rho o-1} \div \iota_{0 t}+\left[\left(1+\varepsilon_{i^{\prime} t}\right)+\varepsilon_{s r t}\right]^{\rho o}
$$

Llegamos a una ley que no puede tener más restricciones que las introducidas. Ahora sólo resta instanciarla a partir de la siguiente información: cada proteína de la población de hemoglobina presenta cuatro subunidades $(\rho o=4)$, la afinidad de la hemoglobina por el monóxido $\left(\kappa_{I}\right)$ es 200 veces mayor que por el oxígeno $\left(\kappa_{S}\right)$ y finalmente seleccionar las cantidades de cada 
ligando en el sistema. Con eso, estaríamos en condiciones de precisar el comportamiento del sistema ICO, pero ¿tenemos pruebas de que las restricciones formalmente introducidas tienen su contraparte empírica?

Las investigaciones de cristalografía de rayos $\mathrm{X}$ de la hemoglobina, que le permitieron ganar el premio Nobel de Química en 1962 a Max Perutz, constituyen un apoyo empírico a nuestra propuesta: la estructura de la oxihemoglobina (hemoglobina unida al oxígeno) no se describió directamente, debido a las dificultades para cristalizarla. Lo que finalmente se cristalizó fue el complejo entre hemoglobina y monóxido de carbono para el estudio de su estructura mediante difracción de rayos X. "Al ser este compuesto un inhibidor competitivo de la unión de oxígeno, la estructura que resulta de su unión es idéntica a la de la oxihemoglobina" (Vera, 2010: 227), es decir, el inhibidor competitivo, como el oxígeno, se une a la conformación R.

Finalmente, completa nuestro análisis, el tratamiento médico para los casos de intoxicación por monóxido de carbono: como es producto de una inhibición competitiva, el mismo consiste en suministrar cantidades mayores de oxígeno para que desplace, por competencia (valga la redundancia), el monóxido de carbono de los sitios de unión de la hemoglobina y se restablezca el suministro de oxígeno a los tejidos.

De la misma forma el sistema COX-ibuprofeno (e incluso la ACTasa-malato) es capturado introduciendo las restricciones nómicas anteriores y mediante el mismo conjunto de leyes especiales. Lo que varía entre estos sistemas es la instanciación final de la (misma) ley terminal.

\subsection{El cambio teórico}

La referencia que se establece en la bibliografía acerca de que es TMWC la que explicaría los fenómenos aquí discutimos y, por otro lado, nuestro análisis de que no resulta así, nos permite suponer que se está pensando en la existencia de una teoría "del tipo de" TMWC (o parecida a esta) que daría cuenta de los fenómenos que están bajo análisis. Esta teoría no es explicitada en los textos, pero de algún modo entendemos que está presupuesta, por lo que podemos pensar además que es de mayor generalidad que las presentadas, e implicada en el abordaje de tales problemas.

Si se concede que en la bibliografía del área hay una teoría implícita y que dicha teoría es como la presentada en el parágrafo anterior como TMMM (o al menos es similar a ella en lo que hace a las características de los conceptos que involucra y la capacidad explicativa), entonces se plantea un ejemplo interesante para el análisis del cambio teórico involucrado y el progreso científico.

La tipología de cambio (I a V) citada más arriba, parte de establecer la génesis del cambio en el surgimiento de alguna entidad nueva (II, III y IV), 
en la explicación de un nuevo fenómeno (I) o en cambios radicales cuya génesis es una combinación de las anteriores (V). Los casos II a IV claramente involucran cambios en los que la génesis es empírica.

En el caso que se expuso en el parágrafo anterior, el origen del cambio parece tener una raíz teórica. Se propuso una teoría más general (TMMM) que permite explicar los fenómenos no explicados por TMM y por TMWC pero que además permite explicar los fenómenos previamente explicados por estas dos teorías, cuyas aplicaciones no coinciden completamente. Tanto TWMC como TMM tienen algún caso de aplicación propio que no lo es de la otra teoría. TMMM permite obtener, con las restricciones adecuadas, las teorías TWMC y la TMM, punto que aún hay que demostrar. Pero si esto último se nos concede, la relación que se establecerá entre TMMM y estas dos teorías es, lo que habitualmente se conoce en la filosofía de la ciencia como, reducción teórica. En ambos casos la manera de obtenerlas no es idéntica. Veamos los casos, pero antes introduciremos, de manera sucinta, las características de este vínculo.

Para que se cumpla la relación de reducción entre dos teorías, llamemos $\mathrm{T}$ a la teoría reducida y $\mathrm{T}^{*}$ a la teoría reductora, se deben dar las siguientes condiciones (presentadas de manera informal): i. tiene que haber una "correspondencia global" entre el marco conceptual de la teoría $\mathrm{T}$ y el de la $\mathrm{T}^{*}$, es decir entre los conjuntos de sus componentes; ii. la correspondencia también se tiene que dar al nivel de las aplicaciones de las teorías, es decir las aplicaciones de $\mathrm{T}$ deberán tener un correlato en $\mathrm{T}^{*}$ pero no necesariamente a la inversa; y iii. siempre que una aplicación cumpla las leyes de la teoría reductora $\mathrm{T}^{*} \mathrm{y}$ además cumpla con las restricciones específicas impuestas por un sistema particular, entonces el correlato de esa aplicación cumplirá también las leyes de la teoría reducida T. Es decir que las leyes generales de la teoría reducida serán implicadas por las leyes generales, más algunas de las leyes especiales, de la reductora. ${ }^{3}$

La reducción a su vez, puede darse de dos maneras, exacta o aproximativa. En el primero de los casos las leyes fundamentales de la teoría reducida se "deducen" (en el sentido lógico) de las leyes generales (y algunas especiales) de la teoría reductora. En el segundo de los casos, las leyes de una y de la otra teoría presentan lenguajes distintos, producto de marcos conceptuales diferentes, pero factibles de alguna clase de "traducción" o correlación.

Como por lo general el análisis de los vínculos interteóricos se suele hacer entre pares de teorías, de forma sistemática comenzaremos por ver bajo qué tipo de reducción (exacta o aproximativa) cae el vinculo entre TMMM y TMM. Mostraremos en primer lugar la forma en que a partir de TMMM se llega a la ley de los sistemas michaelianos: si introducimos la restricción especifica de que la cantidad de proteínas en estado $\mathrm{T}$ es despreciable frente a la cantidad en estado R ( $\iota_{0}$ se hace cero) sobre la segunda ley general de TMMM

\footnotetext{
${ }^{3}$ El desarrollo formal puede verse en Moulines y Diéz, 1997.
} 
(para antes de que haya ligando) y, en la tercera ley general, suponemos la ausencia de moduladores alostéricos $(A$ e $I)$, introducimos además que las afinidades por los ligandos son iguales para ambos estados conformacionales $\left(\varepsilon_{s r t}=\varepsilon_{s t}\right), \mathrm{y}$, finalmente, que las proteínas son simples, es decir, sólo tiene una subunidad $(n=1)$, con todo, la actividad biológica adquiere el siguiente valor específico:

$$
\delta_{(t)}=\varepsilon_{s t t} \cdot\left[\left(1+\varepsilon_{i^{\prime} t}\right)+\varepsilon_{s r t}\left(1+\varepsilon_{i^{\prime \prime} t}\right)\right]^{\rho o-1} \div\left[\left(1+\varepsilon_{i^{\prime} t}\right)+\varepsilon_{s t t} \cdot\left(1+\varepsilon_{i^{\prime \prime} t}\right)\right]^{\rho o}
$$

Operando matemáticamente sobre esta ley (que llamaremos ley especial $\mathrm{MM})$, el valor equivalente que toma es:

$$
\delta_{(t)}=\varepsilon_{s t t} \div\left[\left(1+\varepsilon_{i^{\prime} t}\right)+\varepsilon_{s r t^{*}} \cdot\left(1+\varepsilon_{i^{\prime \prime} t}\right)\right]
$$

que es, en forma, igual a la ley fundamental de la teoría michaeliana. Y como tal es susceptible de seguir especificándose (vía nuevas y distintas restricciones) hasta arribar a las ecuaciones más especializadas de TMM.

Si analizamos los marcos conceptuales (el conjunto de componentes) de cada una de las teorías notaremos que difieren en sus conceptos teóricos: el "estado conformacional" y la función que representa "la conformación en un momento temporal" presentes en TMMM se encuentran ausentes en la propuesta michaeliana. Pero también ocurre que algunos conceptos no teóricos se encuentran ausentes, pues para caracterizar las proteínas michaelianas, al ser estas "mas sencillas", no es necesario introducir el concepto de subunidad, número representado por $r$, que sí lo es para la cooperatividad alostérica y donde además cada subunidad tiene un sitio de unión para cada tipo de ligando, sobre los cuales también difieren, ya que la michaeliana no contempla los moduladores.

Esto indica que entre ambas teorías hay existencia de una ontología parcialmente en común según sus conjuntos base, lo que implica, en algún sentido, que sean teóricamente inconmensurables (por ausencia de conceptos de una a la otra teoría), que hay ausencia de una correspondencia total entre "las bases empíricas" (el conjunto de los modelos de datos) y por tanto entre sus términos no teóricos. La situación que parece se plantea aquí (remitiéndonos a Lorenzano, 2012), es que no todos los términos con problemas de "traducibilidad" se identifiquen con los teóricos, sino que lo hacen con algunos de ellos y con algunos no teóricos. En conclusión, podemos establecer ciertas conexiones entre ambas teorías, pues "hablan de lo mismo", pero la relación entre ellas no es de identidad. Lo que nos invita a pensar que el vínculo entre TMMM y TMM es de reducción aproximativa y, además, la traducción ocurre en el caso límite en que las proteínas con cooperatividad dejan de comportarse como tal. Las características de esta conexión interteórica se asemeja a la existe en algunas aplicaciones físicas entre la relatividad especial de Einstein y la teoría de Newton. Se suele afirmar que la segunda es un caso límite de la primera cuando las velocidades que se consideran en el sistema bajo análisis son despreciables frente a la velocidad de la luz. 
Distinta es la situación que se establece entre TMMM y TMWC. Entre los conceptos MMM no-teóricos se encuentran los MWC- no teóricos y algunos conceptos de $\mathrm{TMM}^{4}$. Es necesario agregar los conceptos teóricos, a saber, los estados conformacionales $\mathrm{T}$ y $\mathrm{R}$ respectivamente, el estado conformacional en que se encuentra la proteína en un momento particular $\xi$ y las constantes de afinidad para cada estado conformacional y cada tipo de sustrato, que son todos los conceptos MWC-teóricos, junto con estos incluimos las constantes de afinidad para cada estado conformacional de los inhibidores no moduladores, conceptos teóricos nuevos ${ }^{5}$. Es factible por tanto, encontrar entre TMMM y TMWC que hay una correspondencia formal entre sus marcos conceptuales, pues los componentes del dominio de TMWC, y sus relaciones, están incluidos en los dominios de TMMM. Pero, como no son idénticos, TMMM puede tratar algunos sistemas completamente distintos y ajenos a los abarcados por TMWC. Asimismo, las leyes generales de TMWC son "implicadas" por las leyes generales, más algunas especiales adicionales necesarias para la deducción de TMMM (vimos que en TMMM se generaliza aún mas la tercera ley, respecto a TMWC). Finalmente, para que la incorporación sea verdaderamente exitosa debe estar garantizada que todas las aplicaciones exitosas de TMWC queden englobadas por las aplicaciones exitosas de TMMM. Es decir que las aplicaciones de TMWC son reinterpretadas como aplicaciones de TMMM, si bien en el presente trabajo por mor del espacio no vamos a desarrollarlo, el arribar a cada una de ellas no implica más que un ejercicio de lápiz y papel. Lo que nos lleva a pensar que el vínculo entre estas dos teorías es de reducción exacta.

A partir de la elucidación de este ejemplo biológico de cambio interteórico estamos en condiciones de plantear una discusión sobre un caso de cambio progresivo en bioquímica, biología y ciencias biomédicas.

\section{Un ejemplo bioquímico de Progreso científico}

El progreso científico y las características que este tiene, es un tema de debate dentro de la filosofía de la ciencia. Podemos limitar el alcance de esta problemática y remitirnos a cambios progresivos en una determinada disciplina científica o dentro de un área específica de ésta.

En el caso que nos ocupa, la pregunta es si constituye un cambio progresivo la adopción de una teoría como TMMM. Para respondernos tal pregunta haremos uso del instrumental metateórico aportado por corrientes epistemológicas que transitan por el mismo camino: la noción diacrónica de progreso de Philip Kitcher y la estructuralista de explicación de Thomas Bartelbroth. Empezaremos por el primero.

\footnotetext{
${ }^{4}$ Estos conceptos pueden ser presentados como el siguiente conjunto ordenado $\left\langle O, S, A, I, I^{\prime}, I^{\prime \prime}, T, \rho, \sigma, \beta\right.$, $\delta\rangle$ donde $I$, representan los inhibidores moduladores; $I^{\prime}$, los inhibidores competitivos; $I^{\prime \prime}$, los inhibidores no competitivos y el resto de los conceptos que se mantiene igual (mismo significado) que en TMWC.

5 Presentados como un conjunto ordenado, son: $\left\langle O, S, A, I, I^{\prime}, I^{\prime \prime}, T,\{\tau, r\}, \rho, \sigma, \beta, \delta, \zeta, \kappa_{S}, \kappa_{A}, \kappa_{I^{\prime}}, \kappa_{l^{\prime}}, \kappa_{I^{\prime \prime}}\right\rangle$.
} 
P. Kitcher plantea que un aspecto que interviene en la determinación del progreso científico es el que llama "progreso explicativo". El progreso explicativo a su vez se asocia con el modelo de explicación científica que propone. Este modelo es conocido en la bibliografía sobre el tema como un modelo de unificación. Lo que resulta relevante para hablar de progreso explicativo, dejando de lado los detalles técnicos de su propuesta, es que, en el devenir del proceso se avance en patrones explicativos que permitan unificación, es decir que se logre dar cuenta de un mayor número de fenómenos con un menor número de patrones.

T. Bartelbroth hace propia esta idea de unificación para rescatar las características de las explicaciones científicas:

Una característica esencial de una buena explicación tal vez se puede ver en un ejemplo sencillo de Philip Kitcher. Explicación, en mi opinión, consiste principalmente en una unificación de la mayor cantidad posible de fenómenos por una teoría. Esta tiene que señalar la estructura en común de muchos eventos que es responsable de ciertas propiedades de estos eventos. En algunos casos esta estructura es causal, pero en otros casos es de otro tipo (Bartelborth, 1996: 214).

En tal sentido entendemos que "la explicación" consiste principalmente en unificar la mayor cantidad, como sea posible, de fenómenos (y de leyes) bajo una misma teoría:

Una explicación de un acontecimiento singular, que es descrito por un modelo de tipo parcial es, desde este punto de vista, una incrustación (formal) de un modelo "pequeño" en un modelo "grande" que obedece a las leyes de la teoría. Por supuesto, no toda incrustación puede considerarse explicativa. T tiene que cumplir con ciertas restricciones adicionales (Bartelborth, 1996: 215).

Es decir, en la versión kitcheriana, el poder de unificación de una teoría (o esquema explicativo) reside en explicar más fenómenos con menos patrones explicativos: una buena teoría debería unificar los datos y las leyes de los diferentes dominios empíricos. Un caso paradigmático es la unificación exitosa de las leyes del movimiento planetario de Kepler y las leyes del movimiento de Galileo a través de la teoría de Newton (Kitcher 1993).

Retomando la noción de progreso científico los campos de la ciencia avanzan explicativamente cuando las prácticas posteriores introducen esquemas explicativos "mejores" que los adoptados por las prácticas anteriores (Kitcher, 1993: 106). "Mejorar" tales esquemas consiste, según el autor, en la adecuación de nuestros esquemas al ordenamiento de los fenómenos (la versión realista robusta) o en la producción de esquemas que son más capaces de cumplir algún criterio de organización, por ejemplo, una mayor unificación. Nos quedamos con éste último. 
El punto fundamental, sin embargo, es que cualquiera de estas formas de definir una noción de progresividad depende de una noción antecedente de progresividad como una relación binaria entre las prácticas. Digamos que la secuencia de las prácticas $\mathrm{P}_{1}, \ldots, \mathrm{P}_{\mathrm{n}}$ es ampliamente progresivo sólo en caso de que para cada par de elementos adyacentes haya un componente de la práctica con respecto a la cual el cambio de la anterior a la posterior es progresiva y el cambio de $\mathrm{P}_{1}$ a $\mathrm{P}_{\mathrm{n}}$ es progresiva con respecto a cada componente de la práctica (Kitcher, 1993: p.92).

Así, para que el progreso científico ocurra, entendido como relación binaria, se tiene que dar alguna de las siguientes situaciones: ${ }^{6}$ i. que las practicas posteriores contengan esquemas explicativos correctos mientras que practicas anteriores no o, ii. que las prácticas posteriores contengan esquemas explicativos más generales (tratar una clase más amplia de casos) que extiendan, o completen, los esquemas de las prácticas anteriores (más aplicaciones exitosas de distinto tipo) o, iii. que las practicas posteriores presentan "extensión explicativa" (que los esquemas se insertan dentro de un esquema más amplio) respecto a las prácticas anteriores (lo que usualmente se entiende por reducción).

El segundo de los casos lo podemos reinterpretar como cambio intrateórico, es decir, de evolución teórica. mientras que el primero y el tercero se reconceptualizan como cambios interteóricos. La primera de las situaciones es la de suplantación o revolución científica y la tercera es la de incorporación. En palabras del autor:

Cuando en mis ejemplos parece haber procesos acumulativos especificables se introducen esquemas refinados, generalizándose y extendiéndose. Pero también abarcan episodios "revolucionarios" y episodios "normales" [...] Por otra parte, si se quiere extender la noción de "ciencia normal" (o algún equivalente) para cubrir la totalidad de los períodos a través del cual traza el refinamiento, la generalización y extensión de los esquemas, entonces será

\footnotetext{
${ }^{6}$ Cuatro tipos distintos de procesos se rescatan en estos ejemplos de progreso explicativo. En primer lugar, tenemos la introducción de esquemas correctos, ilustrado por el trabajo de Dalton en el reconocimiento de la dependencia de los hechos sobre el curso de las reacciones químicas (en concreto sobre los pesos de reactivos y productos) en los hechos acerca de combinación atómica y por una visión de Darwin de que las distribuciones y las relaciones entre los organismos contemporáneos dependen del curso de la descendencia con modificación. En segundo lugar, tenemos la eliminación de esquemas incorrectos, tales como las apelaciones de Darwin a la herencia de los caracteres adquiridos. En tercer lugar, nos encontramos con la generalización de esquemas, haciéndolos capaces de tratar correctamente con una clase más amplia de casos: los teóricos evolutivos que apelan a la selección individual clásica solos son correctas en la identificación de un cierto tipo de dependencia, pero sus propuestas son menos general y, por tanto, menos completa, de las que permite la deriva, la migración, la unidad de la meiosis, los efectos de la aptitud inclusiva, las limitaciones del desarrollo, y así sucesivamente. Por último, está la extensión explicativa, cuando la imagen de las dependencias se inserta dentro de un esquema más amplio. La incorporación de patrones seleccionistas de Darwin dentro de la selección neodarwinista y la incorporación de la química atómica en la física cuántica demuestran este proceso en el trabajo (Kitcher, 1993: 109-110).
} 
necesario minimizar la importancia de las "revoluciones" en la ciencia. Estos períodos son tan grandes, y los procesos que identifican tan frecuente, que la "ciencia normal" estará en todas partes una vez que un campo alcanza la madurez (Kitcher, 1993: 92).

En síntesis, si se concede la suposición de que existe en la bibliografía de la bioquímica, biología y ciencias biomédicas una teoría implícita como la aquí precisada, la que llamamos TMMM, entonces bajo el concepto de progreso kitcheriano podemos sostener que esta teoría implica un progreso en el área ya que explica los sistemas capturados por TMM, los capturados por TMWC más sistemas que escapan a estos como COX e ICO (y ATCasa). Además, parece ocurrir que TMMM trae consigo un progreso explicativo de tipo iii (de los mencionados en el párrafo anterior) respecto de TMWC y un progreso de tipo ii respecto de la TMM.

Si nos remitimos a la tipología de cambio presentada en el parágrafo 2, el tipo de cambio analizado correspondería a uno de los mencionados como de tipo III.

Una pregunta que queda por responder es si efectivamente los científicos buscan el progreso explicativo que se mostró arriba. Dicho de otro modo, si los científicos buscan explicaciones unificadoras o si simplemente se contentan con disponer de modelos que den cuenta de los fenómenos sin que necesariamente se intente llegar a minimizar el número de patrones explicativos. Sin pretender generalizar, el caso presentado parece mostrar que no es así, pues a pesar de estar disponible la teoría unificadora (TMMM) no parece ser su búsqueda parte prioritaria en la agenda del área o al menos su explicitación en la bibliografía habitual. No es nuestro interés responder esta pregunta aquí pero sí llamar la atención acerca del problema. Los filósofos de la ciencia proponen ideales respecto de la búsqueda de explicaciones que la historia de la ciencia parece no refrendar?

\section{Conclusión}

En el presente trabajo hemos mostrado un caso de cambio teórico en bioquímica que nos permite avanzar en el análisis del progreso científico en esta área de conocimiento , como también analizar la adecuación de los modelos propuestos de cambios teóricos de la filosofía en un campo particular de la ciencia. Podemos concluir a partir de nuestro trabajo que:

1. una teoría que generalice los casos de THMM y TMWC no puede constituirse con la simple unión de modelos ya que existe cierta inconmensurabilidad entre ambas;

\footnotetext{
${ }^{7}$ Uno de los autores de este artículo abordó parcialmente este problema mostrando un ejemplo en el que la historia no iba de la mano con lo propuesto por Wesley Salmon (Salmon, 1990; 1995). (Ver Paruelo y Miguel, 2006 y De Regt, 2006).
} 
2. la teoría TMMM, propuesta como reductora de la teoría TMWC, da cuenta de todas las aplicaciones de dicha teoría y también de las de THMM. Esto justifica que se afirme que TMMM es una genuina teoría que además da cuenta de casos no abarcados por las teorías anteriores (sistema COX, ICO y ACTasa), y

3. la adopción de TMMM lleva consigo progreso explicativo y por lo tanto progreso científico de acuerdo a ciertas concepciones de progreso como la de P. Kitcher.

Creemos que nuestra presentación es un aporte más al conocimiento de ambas áreas disciplinares.

\section{Referencias bibliográficas}

Alleva, Karina; Diez, José Antonio y Federico, Lucía (2012): "La teoría MWC (Monod, Wyman y Changeux): El sistema alostérico" en Ágora, Papeles de Filosofía, Monográfico "Nuevas contribuciones iberoamericanas a la Metateoría Estructuralista", No 31(2):225-250.

BARTELBORTH, Thomas (1999): "Coherence and explanations" en Erkenntnis, $\mathrm{N}^{\mathrm{o}}$ 50:209-224.

De Regt, Henk (2006): "Wesley Salmon's Complementarity Thesis: Causalism and Unificationism Reconciled?" en International Studies in the Philosophy of Science, $\mathrm{N}^{\mathrm{0}}$ 20(2):129-147.

Diez, José Antonio (2002): "Explicación, unificación y subsunción teórica". En Wenceslao González (ed.), Pluralidad de la explicación científica. Barcelona: Ariel, pp. 73-93.

Diéz, José Antonio y Moulines, Carlos Ulises (1997): Fundamentos de filosofía de la ciencia. Barcelona: Ariel, S.A.

Kesse, Amit y Ben-Tal, Nir (2011): Introduction to Proteins: Structure, Function, and Motion. Chapman \& Hall/CRC Mathematical and Computational Biology Series. Boca Raton: CRC Press.

Kitcher, Philip (1993): The Advancement of Science. Oxford University Press.

LoRenZANo, Pablo (2012): "Estructura y explicaciones intencionales, inconmensurabilidad teórica y comparabilidad empírica en la historia de la genética clasica" en Lorenzano, Pablo y Oscar Nudler (eds). El camino desde Kuhn: la inconmensurabilidad hoy. Madrid: Biblioteca Nueva.

Miguel, Hernán; Paruelo, Jorge y Pissinis, Gillermo (2002): "Las salvedades (provisos) y la magnitud del cambio teórico" en Crítica: Revista Hispanoamericana de Filosofia, № 34(101):43-71.

Miguel, Hernán y Paruelo, Jorge (2006): "Coexistencia pacífica de explicaciones y reducción" en Revista de Filosofia. №31(2):155-172. 
Salmon, Wesley (1990): "Scientific Explanation: Causation and Unification" en Crítica, Revista Hispanoamericana de Filosofia. No 66:3-23.

Salmon, Wesley (1995): "La comprensión científica en el siglo veinte" en Revista Latinoamericana de Filosofía, No 21(1):3-22.

VERA, Luis Franco (2010): "La hemoglobina: una molécula prodigiosa" en Revista de la Real Academia de Ciencias Exactas Físicas y Naturales. $\mathrm{N}^{\mathrm{o}}$ 104(1):213-232.

WWPDB: Wordwide Protein Data Bank. Consulta 20 de Marzo del 2016: https://www.wwpdb.org/

Zou, Hechang et al. (2012): "Human cyclooxygenase-1 activity and its responses to COX inhibitors are allosterically regulated by nonsubstrate fatty acids" en Journal of Lipid Research, № 53(7):1336-1347. 